\title{
HEAD AND NECK
}

\section{Differential isoform expression of SERCA and myosin heavy chain in hypopharyngeal muscles}

\section{Espressione differenziale di isoforma del SERCA e delle catene pesanti della miosina nella muscolatura ipofaringea}

\author{
MOHAMMED ELRABIE AHMED ${ }^{12}$, H. BANDO' ${ }^{1}$, S. FUSE ${ }^{1}$, H. MOSTAFA ABDELFATTAH ${ }^{3}$, \\ MONA ELRABIE AHMED ${ }^{4}$, M. ABDEL-KADER AHMED ${ }^{2}$, T. TSUJIKAWA ${ }^{1}$, Y. HISA ${ }^{15}$ \\ ${ }^{1}$ Department of Otolaryngology-Head and Neck Surgery, Kyoto Prefectural University of Medicine, Kyoto, Japan; \\ 2 Department of Otorhinolaryngology-Head and Neck Surgery, Sohag University Hospitals, Faculty of Medicine, \\ Sohag University, Sohag, Egypt; ${ }^{3}$ Department of Otorhinolaryngology-Head and Neck Surgery, Alexandria University, \\ Alexandria, Egypt; ${ }^{4}$ Unit of Phoniatrics-Department of Otorhinolaryngology-Head and Neck Surgery, Sohag \\ University, Egypt; ${ }^{5}$ Department of Speech and Hearing Sciences and Disorders, Kyoto Gakuen University, Japan
}

\begin{abstract}
SUMMARY
Composition of slow, fast and hybrid fibres of pharyngeal muscles, associated with pharyngeal movements and regulation, has been rarely studied. The present study aimed to identify expression of sarcoplasmic reticulum Ca2 ${ }^{+}$ATPase (SERCA) and myosin heavy chain (MHC) and hybrid isoforms in different pharyngeal muscles of young and aged rats as well as humans. Isoform expression profiles of SERCA, MHC and hybrid isoforms among six components of pharyngeal muscle were immunohistochemically evaluated in rat and human. The result showed that pharyngeal muscles predominantly expressed fast fibres (SERCA1 and MHCII), whereas expression of slow fibres (SERCA2 and MHCI) was low, but different depending on muscle components. Inner layer of pharyngeal muscles expressed more SERCA2 and hybrid fibres than the outer layer. Pharyngeal muscles in aged rats showed increased hybrid fibers and SERCA2. Human thyropharyngeus also showed a higher portion of fast fibres compared to cricopharyngeus. Thus, in contrast to abundance of fast fibres, slow and hybrid fibres are differentially expressed depending on muscle components and layers as well as aging. These results lead to further understanding of coordinated regulation for speech and swallowing. The unique data presented in this study on SERCA isoform expressions in both rats and human suggest an ability to handle calcium changes according functional demands.
\end{abstract}

KEY WORDS: Hypopharynx $・$ SERCA $・$ Myosin heavy chain $\bullet$ Isoform $・$ Aging

\section{RIASSUNTO}

La correlazione fra la funzione faringea e la composizione delle fibre lente, rapide ed ibride della muscolatura faringea è stata scarsamente studiata. Questo lavoro si propone di studiare l'espressione del SERCA, MHC ed isoforme ibride muscolari in topi giovani, di età avanzata ed anche in pazienti attraverso metodiche di immunoistochimica. I risultati evidenziano una maggior espressione nella muscolatura faringea delle fibre muscolari veloci SERCA1 e MHCII. Lo strato interno esprime maggiormente SERCA2 e fibre ibride rispetto allo strato esterno. La muscolatura faringea dei topi di età avanzata mostra un aumento di espressione delle fibre ibride e SERCA2. Le fibre muscolari veloci, lente ed ibride sono pertanto espresse in misura diversa a seconda dei fasci muscolari, strati ed età dei pazienti e potrebbero giocare un ruolo nella coordinazione del linguaggio e deglutizione. Questi dati suggeriscono inoltre una possibile variabilità nello sfruttamento del calcio a seconda delle richieste funzionali.

PAROLE CHIAVE: Ipofaringe $\bullet$ SERCA $\bullet$ Catena pesante della miosina $\bullet$ Isoforma $\bullet$ Invecchiamento

\section{Introduction}

Pharyngeal muscles play a crucial role in the deglutition process by applying the proper pressures required to receive and propel swallowed material (bolus) through the pharynx and upper oesophageal sphincter (UES), and to shape the airway to modulate resonance during voice and speech production ${ }^{1}$. In fact, the pharyngeal stage of swal- lowing is involuntary, and is the most rapid and complex phase in the entire deglutition process that requires bilateral sequenced activation and inhibition of more than 30 pairs of different muscles of the mouth, pharynx, larynx and oesophagus ${ }^{2}$. All these different muscles need to be adaptable to boluses of differing volumes, consistency and rheological characteristics.

Moreover, UES prevents reflux of oesophageal contents 
into the pharynx to guard airway aspiration and prevent air from entering the oesophagus while breathing. It is generally agreed that the cricopharyngeus is a major contributor to the UES (thyropharyngeal (TP) muscle in animals), and the cranial cervical oesophagus also contributes to it in its proximal and distal extents. These muscles have unique rich innervation (1:2-1:6 nerve-to-muscle fibre innervation ratio) compared with limb and extraocular eye muscles, which is important for the fine control required for their highly specialised and complex functions ${ }^{3}$.

Weakness and fatigue of pharyngeal muscles due to aging or any other factor may result in risks of malnutrition and/ or aspiration pneumonia because of either miss-direction into the lungs or retention of pharyngeal residue that poses an increased aspiration risk ${ }^{45}$. Indeed, normal pharyngeal deglutition constitutes the most important element in a safe swallowing process.

For the above reasons, understanding the anatomical, physiological and biological characteristics of pharyngeal muscles and their functions in the deglutition process has widely attracted the attention of scientists for decades. However, much remains unknown with respect to the distributions of the different fibre types in the pharyngeal muscles as well as the different muscle functional behaviours. What can be currently inferred from the available information is that pharyngeal muscles are very different from the extensively studied limb and trunk musculatures, whose main functions are locomotion and posture, in terms of specialization that in turn produces functional differences in contraction times, tension generation, endurance and tuning of movement. A condition that permits pharyngeal muscles to engage in extremely rapid and prolonged contraction, perform highly refined contractions, constant activity even at rest, and has specific aging-related influences ${ }^{67}$.

In fact, muscle fibres have very different structures, histochemistries, biochemistries and physiologies. The functional properties of each muscle are established by the speed, force, sustainability of contraction (endurance) and contraction/relaxation rate. The major proteins responsible for contraction and relaxations in skeletal muscle are sarcoplasmic reticulum $\mathrm{Ca}_{2}{ }^{+}$-ATPase (SERCA) and myosin heavy chain (MHC) ${ }^{89}$. SERCA plays the main role in the muscle contraction/relaxation cycle ${ }^{10}$, while MHC in muscle fibres regulates the contraction force and velocity. Consequently, the difference between contraction and relaxation times of fast and slow muscle fibres is linked to different SERCA and/or MHC isoform expression: fast fibres express SERCA1 and MHCII, whereas slow fibres express SERCA2 and MHCI ${ }^{11}$. Fast muscle fibres (type II) are recruited for short maximal efforts with fast contraction speeds and are fatigued easily. In contrast, slow muscle fibres (type I) have a slow contraction speed and are highly resistant to fatigue. Thus, muscle regions with a high proportion of fast type II fibres facilitate rapid and phasic movements, whereas those with a high proportion of type I fibres are generally involved in postural adjustments ${ }^{12}$. Therefore, the proportions and distribution patterns of MHC- and SERCA-containing fibres in muscle are closely related to muscle functions.

The distribution and role of SERCA and MHC isoforms in pharyngeal muscles are relatively poorly documented. Thus, the present study aimed to identify expression of SERCA, MHC and hybrid isoforms in different pharyngeal muscles of young and aged rats. SERCA isoform expression was also examined in two human pharyngeal muscles, thyropharyngeus (TP) muscle and cricopharyngeus (CP). Because of the lack of available information on the expression and role of SERCA isoforms in pharyngeal muscles, the data obtained in the current study may assist in better understanding of the physiological functions of pharyngeal muscle, age-related remodelling and their effects on pathological conditions.

\section{Materials and methods}

\section{Animal tissue preparation}

Adult male Wistar rats at 8-12 weeks of age and weighing $280-350 \mathrm{~g}$ were used in the current study $(\mathrm{n}=10)$. Rats at 24 months of age were also employed as aged models $(n=4)$ (Shimizu Laboratory Supplies Co., Kyoto, Japan). Animals were anaesthetised by intraperitoneal injection of sodium pentobarbital $(30-60 \mathrm{mg} / \mathrm{kg}$ ) and then fixed with $4 \%$ paraformaldehyde following cardiac perfusion. The pharyngolarynxes were excised immediately and immersed in the same fixative for approximately 12 hours at $4^{\circ} \mathrm{C}$. Paraffin processing of tissues was performed before preparation for microtome sectioning at 8-10 $\mu \mathrm{m}$ thicknesses.

\section{Surgical microscopic examination and haematoxylin and eosin staining}

The anatomy of rat pharyngeal muscles was studied carefully under an operating microscope (ICOM 300) by microdissection. Next, a complete set of serial transverse sections (5-8 $\mu \mathrm{m}$ thick) were obtained and mounted on glass slides. Some of these sets were stained with haematoxylin and eosin for initial close anatomical inspection of the rat pharyngeal muscles.

\section{Immunohistochemistry}

Deparaffinised sections were incubated with $3 \% \mathrm{H}_{2} \mathrm{O}_{2}$ in 
PBS for 10 minutes. After microwave treatment (5 minutes $\times 3$ times at $500 \mathrm{~W}$ in citrate buffer, $\mathrm{pH} 6$ ), the sections were incubated in blocking solution $(0.3 \mathrm{M}$ glycine, $50 \mathrm{mM}$ ammonium chloride, and $1 \%$ bovine serum albumin in PBS) for 30 minutes before incubation with primary antibodies. The sections were then incubated with anti-SERCA1 or 2 and anti MHCI or II (Table I) antibodies for 12 days at $4^{\circ} \mathrm{C}$, washed, and incubated with Alexa Fluor $^{\circledR} 488$ donkey anti-mouse IgG and Alexa Fluor ${ }^{\circledR} 594$ donkey anti-goat IgG secondary antibodies for 1 hour at room temperature. Double immunohistochemistry was performed using anti-SERCA1 + anti-SERCA2, and anti-SERCA2 + anti-MHCII antibody combinations for dual detection. Negative controls (no application of the primary antibody) were used to document the extent of non-specific binding of the secondary antibody.

At the beginning of our research, we tried to perform a double IHC for MHC and SERCA study. For, MHC isoforms in frozen section (primary AB I "BA-D5"; IIa "SC-71"; IIb "BF-F3" and IIX "6H1" with secondary AB Alexa 488 IgG2b; Alexa 647 IgG1; Alexa 488 IgM; Alexa 647 IgG1 and Alexa 555 IgM respectively) but, we failed to get immunoreaction at several concentrations. Hence, we shifted to paraffin sections, the current antibodies and protocol. We tried triple IHC and experienced some difficulties for autofluorescence and poor identification of low amounts of expression of MHCI.

Immunostained tissue sections were examined using a FV-1000 laser confocal microscope (Olympus, Tokyo, Japan). Image J 1.46 software was used to manually count and calculate the ratio of cells positive for each antibody.

\section{Human samples}

Human TP and CP muscle specimens were used in the current study. Specimens were intra-operatively obtained from non-malignant laryngeal tissue of patients undergoing total laryngectomy for malignancy if the sampling site was not affected by malignancy or preoperative radiotherapy and specimen sampling did not interfere with diagnosis.

The study involved five male patients with a mean age of 52.7 years. All tissue samples were fixed immediately after surgery in $4 \%$ paraformaldehyde and then embedded in paraffin. Paraffin processing of all specimens and microtome sectioning at 8-10 $\mu \mathrm{m}$ was performed. Similar to rats, standard immunofluorescence staining procedures were applied to evaluate SERCA1, SERCA2 and dual expression in human $\mathrm{TP}$ and $\mathrm{CP}$ muscles. Immunostained tissue sections were examined using a FV-1000 laser confocal microscope, followed by quantification of positive cells for each antibody by ImageJ 1.46 software.

\section{Statistical analysis}

Data of the positive cell ratio for each antibody in rats and humans were statistically analysed using SPSS statistical software version 16. The unpaired Student's t-test was applied to compare differences between SERCA, MHC, and hybrid isoforms in various pharyngeal muscles of young and aged rats as well as humans. Values are presented as means \pm standard deviation (SD). For all statistical tests, $\mathrm{p}<0.05$ was considered as significant.

\section{Results}

\section{Anatomy of rat pharyngeal muscles}

The anatomy of rat pharyngeal muscles was microscopically studied by microdissection (see Fig. 1). As reported by Kobler et al. ${ }^{13}$, rigorous examination of pharyngeal muscle anatomy confirmed that the HP and TP muscles formed the main bulk of the rat posterior pharyngeal wall, which originated from the hyoid bone and thyroid cartilage, respectively, while both muscles inserted obliquely into the midline pharyngeal raphe. HP muscle is not defined in human and higher vertebrate animals and appears anatomically homologous to the superior and middle constrictor muscle. In addition, at the pharyngo-oesophageal junction, we observed muscle fibres that arched across the posterior pharyngeal wall to the opposite side without inserting into the midline raphe. These fibres were overlapped by the more oblique fibres of the TP muscle. According to the previous report, this muscle should be labelled as semicircular SC muscle that does not have an insertion on the cricoid cartilage through serial sections. Thus, it appeared that the rat did not have a strictly defined CP muscle, although the SC resembled it in terms of the general location and arrangement.

Table I. Antibodies used in this study.

\begin{tabular}{lll} 
Isoform & Antibody & Description \\
SERCA1 & VE121G9; ab2819 & Mouse monoclonal to SERCA1 lgG1 (abcam) \\
SERCA2 & SERCA 2 (C-20):Sc-8094 & Goat polyclonal against peptide mapping at C-terminus of SERCA 2 (Santa Cruz Biotechnology) \\
MHCl & NOQ7.5.4D & Mouse monoclonal to slow myosin skeletal muscle lgG1 (abcam) \\
MHCll & MY-32; ab51263 & Mouse monoclonal to fast myosin skeletal muscle lgG1(abcam) \\
\hline
\end{tabular}


Immunolocalisation of myosin and SERCA isoforms in young rat pharyngeal muscle

Expression of myosin (MHCI and II) and SERCA (1 and 2 ) isoforms and hybrid isoforms were evaluated in the six selected pharyngeal muscle components in young rats. The studied hypopharyngeal muscle components were hyopharyngus (HP), thyropharyngeus (TP), semicircular (SC) muscles, palatopharyngeus (PP), soft palate and cervical oesophagus (CE). SERCA1 and MHCII, which are considered as fast fibres, were predominant and similarly expressed in all hypopharyngeal muscles, compared with slow muscle fibres (MHCI or SERCA2) (Fig. 2, Table II). Expression of slow fibres was basically low in all examined muscles, but slow fibres showed differential patterns depending on muscle components, where SERCA2 expression was found in all muscles in high proportion than the corresponding slow MHCI which was observed only in HP, TP and SC (Table II). Hybrid fibres (SERCA1 + 2 or MHCII + SERCA2) were detected in all pharyngeal muscles which may assist their complex functional demands. SERCA/MHC mismatches (mainly SERCA2/ MHCI; HP, $12.9 \pm 1.7 / 6.2 \pm 1$; TP, $14.3 \pm 2.3 / 5.5 \pm 1.6$; SC, $30.9 \pm 2.7 / 9.2 \pm 1.4$ ) were found in normal rats, which usually occur in some pathological muscle diseases such as muscle disuse and denervation. This suggests that calcium handling fibre types are regulated differently than that for myosin (Table II). Furthermore, we evaluated potential differences of SERCA isoforms in muscular layers, comparing inner submucosal layer versus outer layer in $\mathrm{SC}$ and $\mathrm{CE}$ muscles. The inner layer expressed more SERCA2 and hybrid fibres than the outer layer, although sharp delineation between the two layers was indistinct (Fig. 3). Those results indicate that the vast majority of pharyngeal muscles were regulated by fast fibres, together with differentially coordinated distribution of slow and hybrid fibres in pharyngeal muscle components as well as muscular layers, which may assist their complex functional demands in pharyngeal kinetics.

\section{Aging-related changes in pharyngeal muscles}

To understand potential effects of aging on pharyngeal muscles, we performed comparative analysis of MHC and SERCA, and hybrid isoforms in young and aged rats. We found that aged rats showed higher ratios of hybrid fibres in all pharyngeal muscle components than young rats (Table II). Among slow fibres, SERCA2 was increased during aging, whereas MHCI was decreased (Fig. 4 and Table II). These observations suggest the presence of aging-related changes in pharyngeal muscles, where an increase of hybrid fibres and differential slow fibre isoforms was observed.

\section{SERCA expression in human hypopharyngeal muscles}

Finally, we verified our findings in rats by evaluating human tissues derived from clinical specimens by immunohistochemistry. SERCA isoforms were analysed in $\mathrm{CP}$ muscles, in consideration of anatomical differences between human and rat since rats possess SC, but not a counterpart corresponding to $\mathrm{CP}$ in humans. SERCA1
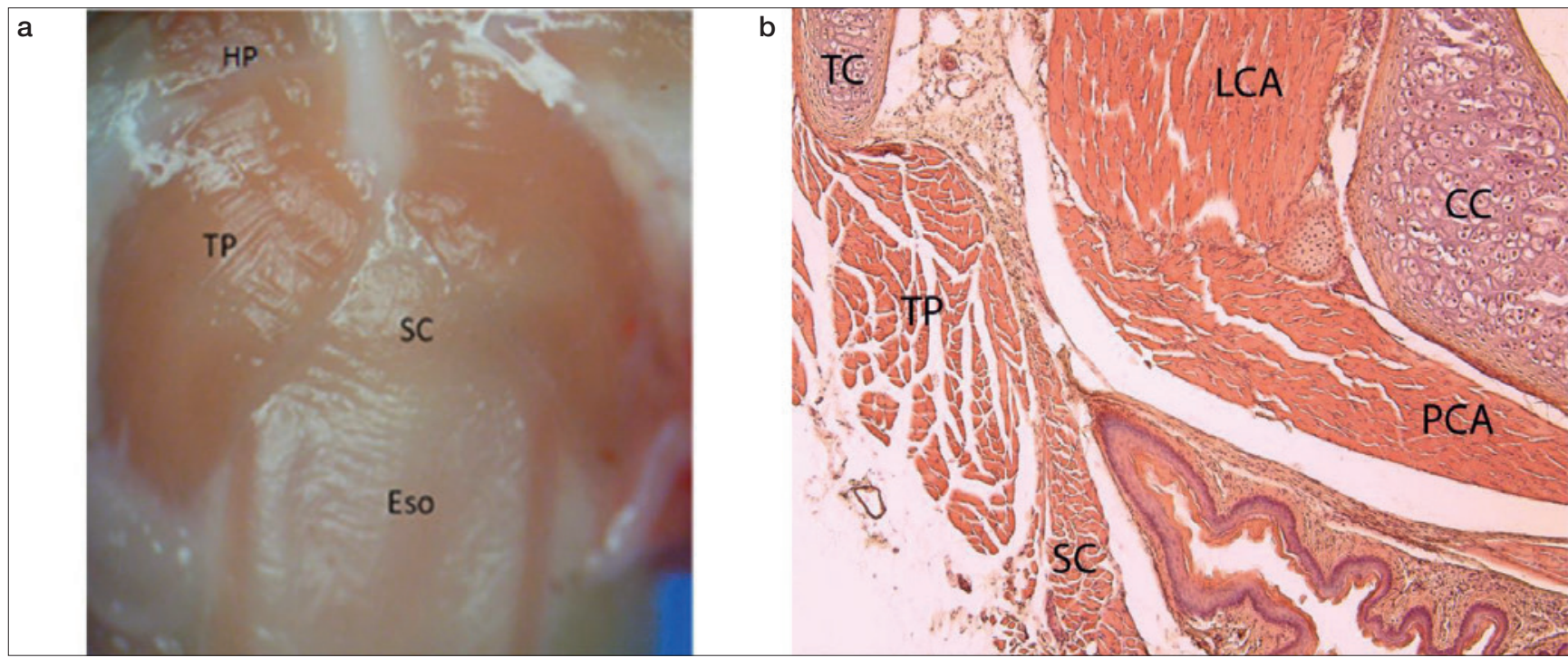

Fig. 1. Anatomical findings of rat pharyngeal muscles. (a) Posterior view of the pharynx showing hyopharyngus (HP), thyropharyngeal (TP) and semicircular (SC) muscles, and the cervical oesophagus (Eos). (b) Haematoxylin and eosin staining ( $\times 200)$ showing SC muscle attachment to thyroid cartilage (TC), LCA (lateral cricoarytenoid), and PCA (posterior cricoarytenoid). 


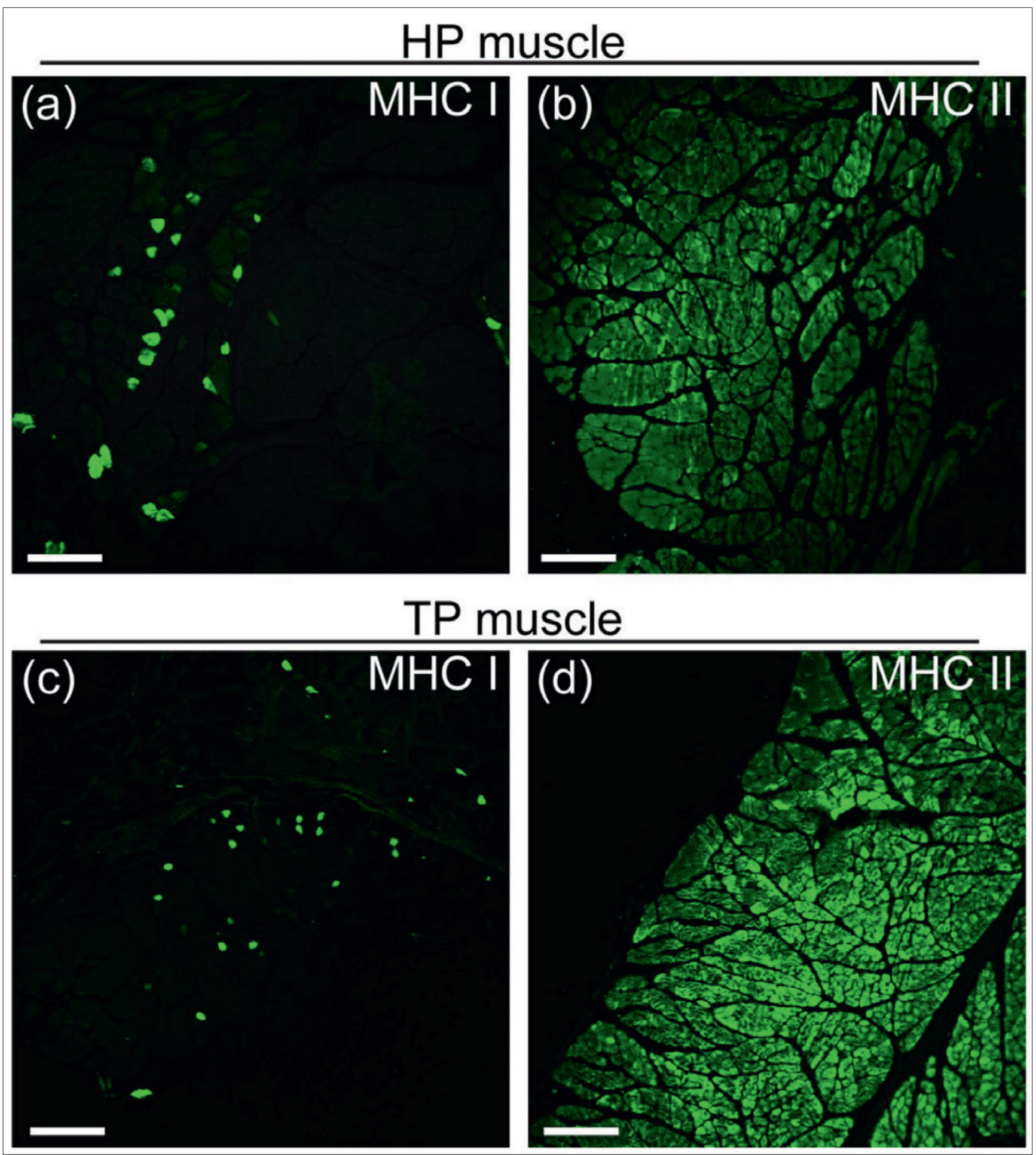

Fig. 2. Myosin heavy chain (MHC) isoform expression in hyopharyngus (HP) and thyropharyngeal (TP) muscles. (a) MHCl expression (slow fibres) in HP muscle, (b) MHCll (fast fibres) in HP muscle, (c) MHCl expression (slow fibres) in TP muscle, and (d) MHCll (fast fibres) in TP muscle; young rats, $\times 100$. Scale bar $=50 \mu$ m.

was less predominant in human $\mathrm{CP}$ muscle in comparison with human TP muscle $(74 \pm 10.1$ in TP vs $47.7 \pm 14.5$ in $\mathrm{CP}$ ). However, human $\mathrm{CP}$ muscle contained more
SERCA2 $(61.9 \pm 14.9)$ and hybrid fibres $(15.6 \pm 3.3)$ than human TP muscle (Fig. 5a and Table III). Next, we analysed isoform expression in TP muscles in which humans 
Table II. Summary of the ratios of cells positive (mean \pm SD) for SERCA1, SERCA2, MHCl, MHCll and hybrids in young and aged rat hypopharyngeal muscles and the results of unpaired t-tests.

\begin{tabular}{|c|c|c|c|c|c|c|}
\hline & SERCA1 & SERCA2 & $\mathrm{MHCl}$ & MHCII & Hybrid (S1+S2) & Hybrid (MII+S2) \\
\hline \multicolumn{7}{|l|}{ Hyopharyngus } \\
\hline Young (mean \pm SD) & $94 \pm 0.4$ & $12.9 \pm 1.7$ & $6.2 \pm 1$ & $94.5 \pm 1$ & $10.4 \pm 4$ & $11.5 \pm 2.9$ \\
\hline Aged (mean \pm SD) & $97.9 \pm 0.7$ & $17.8 \pm 2.2$ & $4.8 \pm 1.7$ & $97.5 \pm 0.6$ & $16.7 \pm 0.8$ & $18.3 \pm 0.6$ \\
\hline Young vs aged ( $P$ value) & $<0.05$ & $<0.05$ & NS & $<0.05$ & $<0.05$ & $<0.05$ \\
\hline \multicolumn{7}{|l|}{ Thyropharyngus } \\
\hline Young (mean \pm SD) & $95.4 \pm 1$ & $14.3 \pm 2.3$ & $5.5 \pm 1.6$ & $94.9 \pm 1.2$ & $12 \pm 2.7$ & $12 \pm 2.2$ \\
\hline Aged (mean \pm SD) & $97.9 \pm 1$ & $16.9 \pm 1.5$ & $3.6 \pm 0.3$ & $98.1 \pm 1$ & $14 \pm 2$ & $14.3 \pm 2.4$ \\
\hline Young vs aged (P value) & $<0.05$ & $<0.05$ & $<0.05$ & $<0.05$ & $<0.05$ & $<0.05$ \\
\hline \multicolumn{7}{|l|}{ Semicircular muscle } \\
\hline Young (mean \pm SD) & $90.7 \pm 1.4$ & $30.9 \pm 2.7$ & $9.2 \pm 1.4$ & $91 \pm 1.4$ & $19.7 \pm 2.7$ & $17.4 \pm 2.8$ \\
\hline Aged (mean \pm SD) & $92.7 \pm 1.9$ & $34.5 \pm 2.5$ & $6.6 \pm 1.5$ & $94.5 \pm 0.9$ & $25.4 \pm 1$ & $26.3 \pm 6$ \\
\hline Young vs aged ( $P$ value) & NS & $<0.05$ & $<0.05$ & $<0.05$ & $<0.05$ & $<0.05$ \\
\hline \multicolumn{7}{|l|}{ Cervical oesophagus } \\
\hline Young (mean \pm SD) & 100 & $26.1 \pm 2.5$ & 0 & 100 & - & - \\
\hline Aged (mean \pm SD) & 100 & $30.8 \pm 2.5$ & 0 & 100 & - & - \\
\hline Young vs aged (P value) & NS & $<0.05$ & NS & NS & - & - \\
\hline \multicolumn{7}{|l|}{ Palatopharyngeus } \\
\hline Young (mean \pm SD) & 100 & $19.2 \pm 3.5$ & 0 & 100 & $18 \pm 4.2$ & $18.3 \pm 4.5$ \\
\hline Aged (mean \pm SD) & $99 \pm 0.8$ & $28.4 \pm 2.4$ & $1 \pm 0.9$ & $99.3 \pm 0.7$ & $28.4 \pm 4.2$ & $28.5 \pm 3$ \\
\hline Young vs aged ( $P$ value) & NS & $<0.05$ & NS & NS & $<0.05$ & $<0.05$ \\
\hline \multicolumn{7}{|l|}{ Soft palate } \\
\hline Young (Mean \pm SD) & 100 & $15.7 \pm 2.3$ & 0 & 100 & $15.6 \pm 3$ & $15 \pm 2$ \\
\hline Aged (mean \pm sD) & $99.7 \pm 0.5$ & $19.1 \pm 2.2$ & $0.9 \pm 0.8$ & $99.6 \pm 0.5$ & $19 \pm 1.7$ & $18.8 \pm 2.7$ \\
\hline Young vs aged (P value) & NS & NS & NS & NS & NA & NS \\
\hline
\end{tabular}

$P<0.05$ was considered as statistically significant; SD: standard deviation.
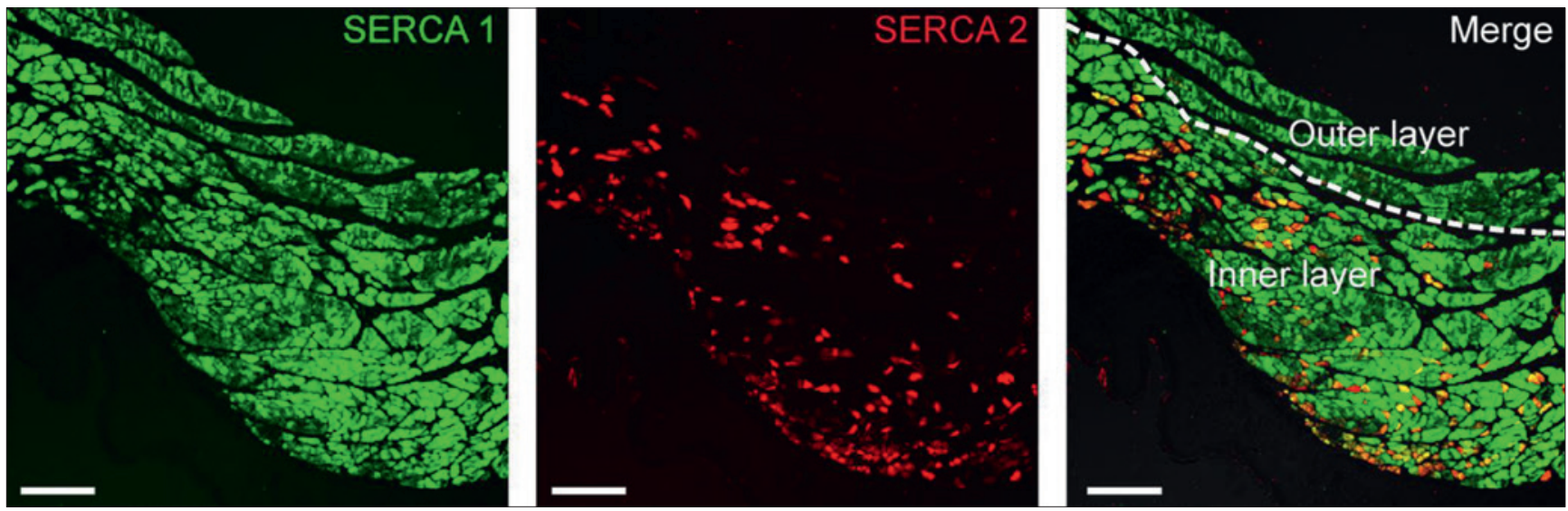

Fig. 3. Differential expression of SERCA isoforms in inner and outer layers of semicircular muscle. SERCA1 (green) was more apparent in the outer layer, while SERCA2 (red) was apparent in the inner layer. Scale bar $=50 \mu \mathrm{m}$.

and rats have anatomical analogy. Human TP muscles showed higher expression of SERCA1 than SERCA2. Those observations were similar to our findings in rats, although SERCA2 was relatively high in human TP muscles (74 \pm 10.1 vs $37.5 \pm 11.8$ ) (Fig. 5 b , Table III), which also leads to the notion that mean age in human samples 


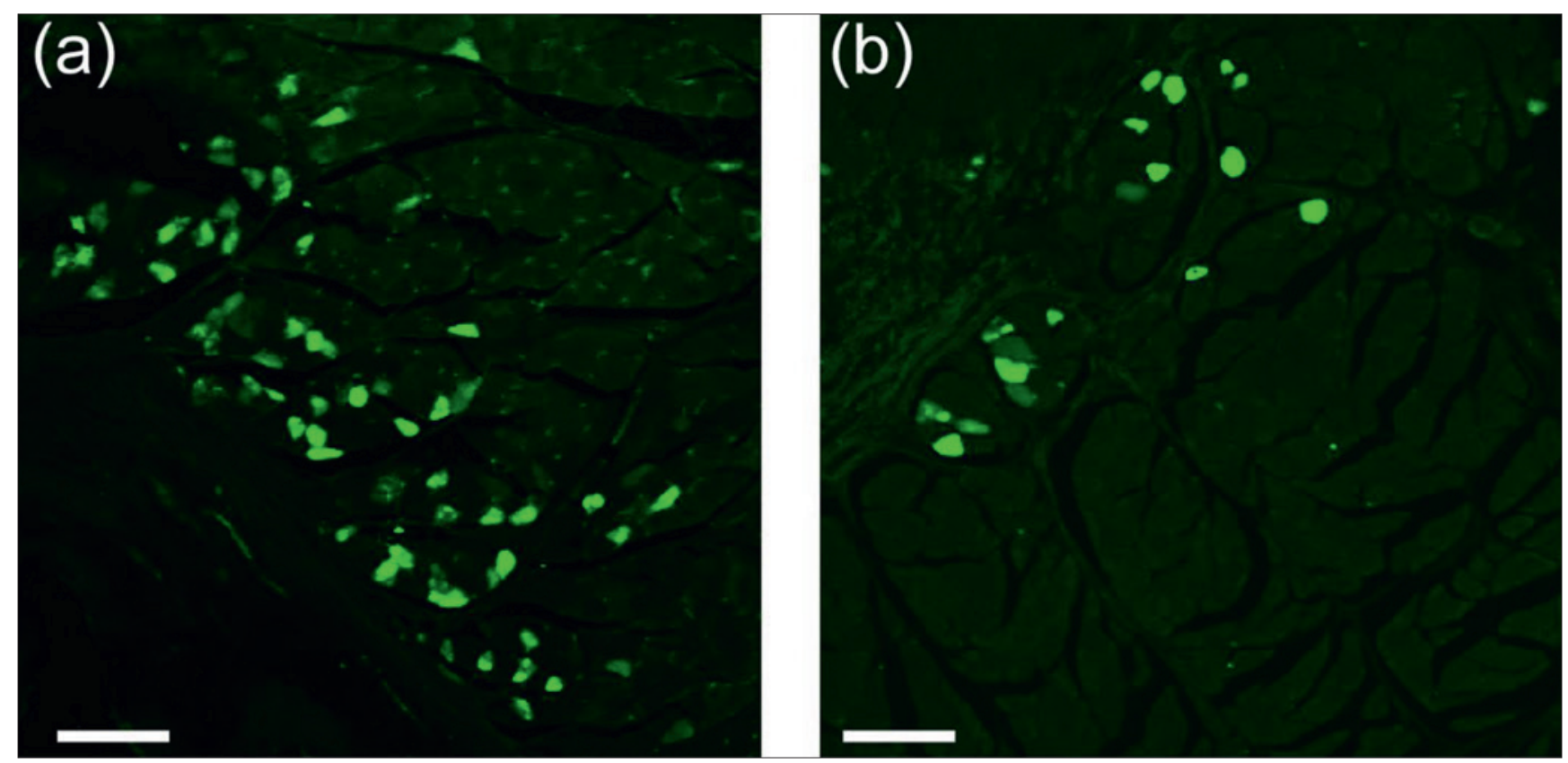

Fig. 4. Age-related decrease of $\mathrm{MHCl}$ in SC muscle: (a) $\mathrm{MHCl}$ in young rats at 8-12 weeks, and (b) $\mathrm{MHCl}$ in aged rats at 24 months. Expression of $\mathrm{MHCl}$ in aged mice decreased in SC muscle. Scale bar $=50 \mu \mathrm{m}$.

is 52.7 years. These data support our observations in rats and highlight differential isoform expression depending on the components of hypopharyngeal muscles.

\section{Discussion}

By expression analysis of SERCA and MHC isoforms in multiple components of pharyngeal muscles, this study has provided further insights into understanding fibre types and their particular functions, which are potentially associated with muscle types and aging. As shown in Table II, all examined pharyngeal muscles predominantly contained fast fibres (SERCA1 and MHCII) at almost similar high ratios (90-100\%), while showing a lower proportion (0-35\%) of SERCA2 and MHCI (slow fibre) expression. SERCA2 was observed at a significantly lower proportion in all muscles, while MHCI was localised only in pharyngeal constrictor (HP and TP) and SC muscles, and not detected in other muscles. However, the rat CE striated muscle was found to contain only fast twitch fibres (MHCII and SERCA1), which is inconsistent with previous observations ${ }^{14}$. In fact, to the best of our knowledge, the present SERCA isoform data represent a unique data set that supplements the few relevant data.

The composition of MHC isoforms in pharyngeal muscle is still controversial. Our results of MHCI isoform profiles supported early data in the literature. Bonington et al. ${ }^{15}$ reported that MHCI accounted for approximately $10 \%$ of the SC muscle, which is similar to the ratio found in the current study. In a study by Taguchi et al. ${ }^{16}$, it was reported that $18.2 \%$ and $37.8 \%$ MHCI were found in TP and SC muscles, respectively, by myosin-ATPase staining, whereas another report showed no detection of $\mathrm{MH}$ $\mathrm{CI}$ in any pharyngeal muscles ${ }^{17}$. These discrepancies are

Table III. Summary of SERCA1, SERCA2, and hybrid (S1+S2) expression values (mean \pm SD) in humans and the results of unpaired t-tests ( $p$-value).

\begin{tabular}{lccc} 
& SERCA1 & SERCA2 & Hybrid (S1+S2) \\
Thyropharyngus (TP) & & & $9.5 \pm 3.9$ \\
Mean \pm SD & $74 \pm 10.1$ & $37.5 \pm 11.8$ & \\
Cricopharyngus (CP) & & & $16.2 \pm 4.1$ \\
Mean \pm SD & $47.7 \pm 14.5$ & $63 \pm 13.1$ & $<0.05$ \\
TP vs CP (P value) & $<0.05$ & $<0.05$ & \\
\hline
\end{tabular}

$P<0.05$ was considered as statistically significant; $S D$ : standard deviation. 
potentially derived from different techniques and a lack of consideration of the anatomical differences in rat hypopharyngeal muscles in which the SC muscle is likely present instead of $\mathrm{CP}$ muscle in upper species including humans (Fig. 1).

Our data of MHC and SERCA expression patterns demonstrated the presence of three different sub-classifications in pharyngeal muscle fibres: fast fibres represented by expression of SERCA1 and MHCII, which are associated with short bursts for rapid and powerful motion despite the lack of endurance; slow fibres represented by expression of SERCA2 and MHCI, which generate fatigue-resistant and sustainable motion with low peak power; hybrid intermediate fibres represented by MHCII, SERCA1, and SERCA2, which have a hybrid phenotype of fast and slow fibres i.e. fast and fatigue-resistant. In fact, muscle regions with a high proportion of fast fibres facilitate rapid, phasic and forceful movements as fast peristaltic contraction that propels the food bolus during swallow. Slow muscle fibres might play a role in maintenance of upper airway patency and prevent the pharyngeal tube from collapse with inspiratory negative pressure. Moreover, hybrid fibres may permit the muscle to recruit the desired form of contraction and relaxation, which allows highly specific fine-tuned movement. This complexity enables organisation of heterogeneous and simultaneous functions during sphincter tonic contraction and relaxation. In addition, the co-existence of both SERCA1 and 2 in either fibre type may be advantageous for more $\mathrm{Ca}^{2+}$-handling versatility ${ }^{18}$. These observations support the hypothesis that the proportions and distribution patterns of MHC and SERCA in muscle fibres are closely related to unique and specific pharyngeal muscle functions.

In this study, we observed interesting histological features in the SC muscle in which fast and slow fibres appeared to divide the muscles into two indistinct layers: a fastouter layer (FOL) containing MHCII/SERCA1 fibres and a slow inner layer (SIL) containing a higher percentage of SERCA2, MHCI, and hybrid fibres (Fig. 3). This property may allow them to function independently and supports the idea that both layers cooperatively generate a shearing effect in the UES. These results are compatible with previous reports indicating that the inner muscle layer with neonatal MHC myofibers is functionally analogous to the slow twitch myofiber layer in humans. Previous studies reported that the human $\mathrm{CP}$, which resembles rat $\mathrm{SC}$ muscle, can also be divided into FOL and SIL fibres ${ }^{19-21}$. $\mathrm{Mu}$ et al. ${ }^{21}$ suggested that the different distributions in SIL and FOL are reflective of their different physiological capabilities and independent functionalities. SIL is associated with fine movements and tonic activity, enabling sustained resting tone of the UES, which is required to prevent aerophagia and occasional forceful contraction during deglutition. FOL is associated with rapid and powerful contraction enabling prompt and phasic dynamics while swallowing. These findings suggest that both fibre
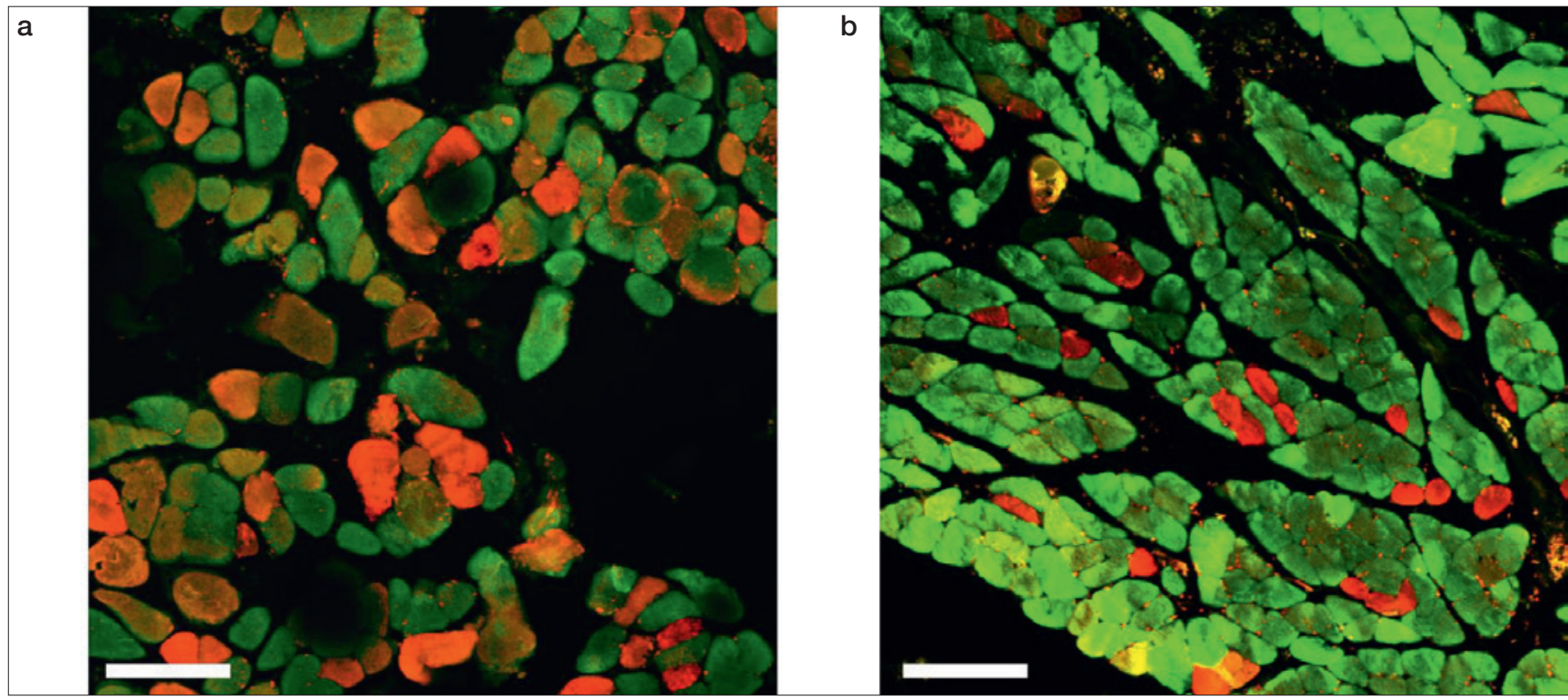

Fig. 5. SERCA isoform expression in human muscles: expression of both SERCA1 (green) and SERCA2 (red) were observed in the cricopharyngeus (CP) muscle (a) and thyropharyngeal (TP) muscle (b). SERCA1 was more predominant in the TP muscle, while SERCA2 was expressed more in the CP muscle. Scale bar $=50 \mu \mathrm{m}$. 
layers have distinct contractile properties and play different roles.

Because muscles are generally susceptible to degeneration- and contraction-induced injuries associated with daily activities, which lead to muscle atrophy and decreased functionality with aging ${ }^{22}$, we investigated the potential aging effects on expression of MHC and SERCA by comparative analysis between young and aged rats. The effect of aging was associated with maintenance of a higher proportion of fast MHCII/SERCA1 fibres. There were also significant increases in SERCA2 and hybrid fibres in all pharyngeal muscles. The age-associated shifts in MHC expression with decrease in MHCI support a muscle-specific change rather than follow a "fast to slow" rule. These changes may be associated with slowing of the contraction-relaxation cycle in pharyngeal muscles. Senescence is associated with loss of swallowing efficiency by slowing of the swallow sensitivity response in the pharynx, coordination of swallowing, impaired opening of the UES and loss of UES elasticity or compliance ${ }^{23}$.

In humans, both SERCA1 and 2 were detected in TP and $\mathrm{CP}$ muscles. Notably, the frequency of slow muscle fibres (SERCA2) in CP muscle was significantly higher than that in TP muscle. This finding is supported by previous reports showing the predominance of MHCI slow twitch fibres in $\mathrm{CP}$ muscle $(>80 \%)^{24} 25$. Our study also showed that $\mathrm{CP}$ muscle had a significantly higher ratio of hybrid fibres than TP muscle. In a similar context, rat SC muscle, which is an analogue to $\mathrm{CP}$ muscle in humans, had the highest frequency of hybrid fibres (SERCA1 and SERCA2) among all examined muscles. These findings support the notion that $\mathrm{CP}$ muscles are relatively biased toward slow and hybrid fibres rather than fast fibres. In fact, TP muscle serves as an inferior pharyngeal constrictor and plays an important role in respiration and maintaining patency of the collapsible pharyngeal airway during swallowing, respiration and vocalisation, which can benefit from the predominance of fast fibres. In contrast, $\mathrm{CP} / \mathrm{SC}$ muscles form a transition sphincter between the pharynx and oesophagus, whereby successive contractions of the pharyngeal constrictors during swallowing propel the bolus downward to the $\mathrm{CP} / \mathrm{SC}$ muscles, followed by its relaxation to allow bolus passage into the oesophagus. Thus, MHCI and SERCA2 myofibers provide the tonic force to maintain closure of the UES, which benefits from the predominance of slow and hybrid fibres. In support of this speculation, slowing of peristalsis and decreased relaxation in the sphincter produce swallowing abnormalities, leading to retained food or saliva in the pharynx after swallowing ${ }^{26}$. Moreover, the variety of hybrid CP/SC muscle fibre types may explain its wide range of contraction characteristics. These observations support that differential compositions of fibre types are associated with differential motor functions of pharyngeal muscles, contributing to coordinated regulation for speech and swallowing movements.

\section{Conclusions}

In this study, we demonstrated different proportions and patterns of slow and fast muscle fibres represented by MHC and SERCA isoforms in pharyngeal muscles in humans and rat. Based on rigorous examination and comparative analyses of the obtained results, the following conclusions can be drawn:

- Pharyngeal muscles have different proportions and patterns of MHC and SERCA, which are potentially associated with coordination of pharyngeal muscle functions.

- Histological analysis of rat SC muscle revealed two distinct layers: SIL and FOL.

- Aging might be associated with an increase of SERCA2 and hybrid fibres in pharyngeal muscles.

- In humans, CP muscle has higher portions of slow muscle (SERCA2) and hybrid (SERCA1 and 2) fibres in comparison with TP muscles.

These observations may provide a new insight into further physiology for regulation of pharyngeal movement and function.

\section{Acknowledgements}

This work was supported by a Grant-in-Aid for Scientific Research (15K1082, 25293350, and 17H07016) from the Japan Society for the Promotion of Science.

\section{Conflict of interest statement}

None declared.

\section{References}

\footnotetext{
$\mathrm{Mu}$ L, Sanders I. Neuromuscular compartments and fiber-type regionalization in the human inferior pharyngeal constrictor muscle. Anat Rec 2001;264:367-77. https://doi.org/10.1002/ar.10020.

2 Cunningham ET, Jones B. Anatomical and physiological overview. In: Jones B, editor. Normal and abnormal swallowing. New York, NY: Springer; 2003. pp. 11-34.

3 Mittal RK. Motor function of the pharynx, the esophagus, and its sphincters. In: Physiology of the gastrointestinal tract. Fifth edition. San Rafael (CA): Morgan \& Claypool Life Sciences; 2011. pp. 91950.

4 Khan A, Carmona R, Traube M. Dysphagia in the elderly. Clin Geriatr Med 2014;30:43-53. https://doi.org/10.1016/j.cger.2013.10.009.

5 Molfenter SM, Steele CM. The relationship between residue and aspiration on the subsequent swallow: an application of the normal-
} 
ized residue ratio scale. Dysphagia 2013;28:494-500. https://doi. org/10.1007/s00455-013-9459-8.

6 Thomas LB. Effect of dystrophin deficiency on selected intrinsic laryngeal muscles of the mdx mouse. University of Kentucky Doctoral Dissertations 2008;591.

7 Vinney LA, Connor NP. Structure and function of the laryngeal and pharyngeal muscles. In: McLoon LK, Andrade F, editors. Craniofacial muscles. New York, NY: Springer; 2012. pp. 141-66.

8 Kjellgren D, Ryan M, Ohlendieck K, et al. Sarco(endo) plasmic reticulum $\mathrm{Ca}^{+}$ATPases (SERCA1 and-2) in human extraocular muscles. Invest Ophthalmol Vis Sci 2003;44:5057-62. https://doi. org/10.1167/iovs.03-0218.

9 Lipskaia L, Chemaly ER, Hadri L, et al. Sarcoplasmic reticulum $\mathrm{Ca}^{+}$ATPase as a therapeutic target for heart failure. Expert Opin Biol Ther 2010;10:29-41. https://doi. org/10.1517/14712590903321462.

10 Periasamy M, Kalyanasundaram A. SERCA pump isoforms: their role in calcium transport and disease. Muscle Nerve 2007;35:43042. https://doi.org/10.1002/mus.20745.

11 Hoh JFY. Laryngeal muscle fibre types. Acta Physiol 2005;183:13349. https://doi.org/10.1111/j.1365-201X.2004.01402.x.

$12 \mathrm{Mu}$ L, Sanders I. Newly revealed cricothyropharyngeus muscle in the human laryngopharynx. Anat Rec 2008;291:927-38. https://doi. org/10.1002/ar.20727.

13 Kobler JB, Datta S, Goyal RK, et al. Innervation of the larynx, pharynx, and upper esophageal sphincter of the rat. J Comp Neurol 1994;349:129-47. https://doi.org/10.1002/cne.903490109.

14 Whitmore I. Oesophageal striated muscle arrangement and histochemical fibre types in guinea-pig, marmoset, macaque and man. $\mathrm{J}$ Anat 1982;134:685-95.

15 Bonington A, Whitmore I, Mahon M. A histological and histochemical study of the cricopharyngeus muscle in the guinea-pig. J Anat 1987;153:151-61.

16 Taguchi A, Hyodo M, Yamagata T, et al. Age-related remodeling of the hypopharyngeal constrictor muscle and its subneural appara- tuses: a scanning electron microscopical study in rats. Dysphagia 2004;19:241-7.

17 Randolph ME, Luo Q, Ho J, et al. Ageing and muscular dystrophy differentially affect murine pharyngeal muscles in a region-dependent manner. J Physiol 2014;592:5301-15. https://doi.org/10.1113/ jphysiol.2014.280420.

18 Fajardo VA, Bombardier E, Vigna C, et al. Co-expression of SERCA isoforms, phospholamban and sarcolipin in human skeletal muscle fibers. PLoS One 2013;12:e84304. https://doi.org/10.1371/journal. pone.0084304.

$19 \mathrm{Mu} \mathrm{L}$, Sanders I. Muscle fiber-type distribution pattern in the human cricopharyngeus muscle. Dysphagia 2002;17:87-96. https:// doi.org/10.1007/s00455-001-0108-2.

$20 \mathrm{Mu} \mathrm{L}$, Sanders I. Neuromuscular specializations within human pharyngeal constrictor muscles. Ann Otol Rhinol Laryngol 2007;116:604-17. https://doi.org/10.1177/000348940711600809.

${ }_{21} \mathrm{Mu} \mathrm{L}, \mathrm{Su} \mathrm{H}$, Wang J, et al. Myosin heavy chain-based fiber types in the adult human cricopharyngeus muscle. Muscle Nerve 2007;35:637-48. https://doi.org/10.1002/mus.20741.

22 Faulkner JA, Brooks SV, Zerba E, et al. Muscle atrophy and weakness with aging: contraction-induced injury as an underlying mechanism. J Gerontol A Biol Sci Med Sci 1995;50 Spec No:124-9. https://doi.org/10.1093/gerona/50a.special_issue.124.

23 McKee GJ, Johnston BT, McBride GB, et al. Does age or sex affect pharyngeal swallowing? Clin Otolaryngol Allied Sci 1998;23:100-6.

24 Bonington A, Mahon M, Whitmore I. A histological and histochemical study of the cricopharyngeus muscle in man. J Anat $1988 ; 156: 27-37$

25 Kristmundsdottir F, Mahon M, Froes MMQ, et al. Histomorphometric and histopathological study of the human cricopharyngeus muscle. Neuropathol Appl Neurobiol 1990;16:461-75.

$26 \mathrm{Mu}$ L, Sobotka S, Chen J, et al. Beach TG Arizona Parkinson's Disease Consortium. Altered pharyngeal muscles in Parkinson disease. J Neuropathol Exp Neurol 2012;71:520-30. https://doi. org/10.1097/NEN.0b013e318258381b.

Received: May 1, 2018 - Accepted: June 12, 2018

How to cite this article: Elrabie Ahmed M, Bando H, Fuse S, et al. Differential isoform expression of SERCA and myosin heavy chain in hypopharyngeal muscles. Acta Otorhinolaryngol Ital 2019;39:220-229. https://doi.org/10.14639/0392-100X-2185

Address for correspondence: Takahiro Tsujikawa, Department of Otolaryngology-Head and Neck Surgery, Kyoto Prefectural University of Medicine, 465 Kajiicho, Kamigyoku, Kyoto City, 602-0841 Kyoto, Japan. Tel. +81-75-251-5603. Fax +81-75-251-5604. E-mail: tu-ji@ koto.kpu-m.ac.jp 